\title{
Asteroseismology of Close Binary Stars
}

\author{
Conny Aerts \\ Instituut voor Sterrenkunde, Celestijnenlaan 200D, B-3001 Leuven, Belgium \\ email: conny@ster.kuleuven.be \\ and \\ Department of Astrophysics, Radboud University Nijmegen, P.O.Box 9010, 6500 GL \\ Nijmegen, The Netherlands
}

\begin{abstract}
In this review paper, we summarise the goals of asteroseismic studies of close binary stars. We first briefly recall the basic principles of asteroseismology, and highlight how the binarity of a star can be an asset, but also a complication, for the interpretation of the stellar oscillations. We discuss a few sample studies of pulsations in close binaries and summarise some case studies. This leads us to conclude that asteroseismology of close binaries is a challenging field of research, but with large potential for the improvement of current stellar structure theory. Finally, we highlight the best observing strategy to make efficient progress in the near future.
\end{abstract}

Keywords. (stars:) binaries: general, (stars:) binaries (including multiple): close, (stars:) binaries: eclipsing, stars: early-type, stars: evolution, stars: interiors, stars: oscillations (including pulsations), (stars:) subdwarfs, stars: variables: other, stars: statistics

\section{Goals and current status of asteroseismology}

The main goal of asteroseismology is to improve the input physics of stellar structure and evolution models by requiring such models to fit observed oscillation frequencies. The latter are a very direct and high-precision probe of the stellar interior, which is otherwise impossible to access. In particular, asteroseismology holds the potential to provide a very accurate stellar age estimate from the properties of the oscillations near the stellar core, whose composition, stratification and extent is captured by the frequency behaviour. In general, the oscillations are characterised by their frequency $\nu$ and their three wavenumbers $(\ell, m, n)$ determining the shape of the eigenfunction (e.g., Unno et al. 1989). Seismic tuning of interior stellar structure becomes within reach when a unique set of values $(\nu, \ell, m, n)$ is assigned to each of the observed oscillation modes. In practice, the number of identified modes needed to improve current structure models depends on the kind of star. For B-type stars on the main sequence, e.g., even two or three well-identified modes can sometimes be sufficient to put constraints on the internal rotation profile (e.g., Aerts et al. 2003, Pamyatnykh et al. 2004) and/or on the extent of the convective core (e.g., Aerts et al. 2006, Mazumdar et al. 2006).

Asteroseismology received a large impetus after the very successful application of the technique to the Sun during the past decade. Helioseismology indeed revolutionised our understanding of the solar structure, including the solar interior rotation and mixing (e.g., Christensen-Dalsgaard 2002 for an extensive review). This remains true today, even though helioseismology is presently undergoing somewhat of a crisis with the revision of the solar abundances (Asplund et al. 2005, and references therein). These led to a slightly diminished precision, but still the relative agreement between the observed solar oscillation properties and those derived from the best solar structure models remains below $0.5 \%$ for most of the basic quantities, such as the interior sound speed and composition profiles (while it used to be below $0.1 \%$ with the old solar abundances). 
Helioseismology thus provided us with a unique calibrator to study the structure of other stars.

However, the Sun is just one simple star. It is a slow rotator, it is hardly evolved, it does not possess a convective core, it does not suffer from severe mass loss, etc. There are thus a number of effects, of great importance for stellar evolution, that have not yet been tested with high accuracy. Since stars of different mass and evolutionary stage have very different structure, we cannot simply extrapolate the solar properties across the whole HR diagram. Stellar oscillations allow us to evaluate our assumptions on the input physics of evolutionary models for stars in which these effects are of appreciable importance. For the moment, such oscillations are the only accurate probe of the interior physics that we have available.

At present, the interior mixing processes in stars are often described by parametrised laws, such as the time-independent mixing length formulation for convection. Values near the solar ones for the mixing length and the convective overshoot are often assumed, for lack of better information. Similarly, rotation is either not included or with an assumed rotation law in stellar models, while an accurate description of rotational mixing is crucial for the evolution of massive stars (e.g., Maeder \& Meynet 2000).

Fortunately, both core overshoot and rotation modify the frequencies of the star's oscillations and they do it in a different way. Core overshoot values can be derived from zonal oscillation modes, which have $m=0$, because they are not affected by the rotation of the star for cases where the centrifugal force can be ignored, but they are strongly affected if an overshoot region surrounds the well-mixed convective core. On the other hand, the rotational splitting of the oscillation frequencies is dependent of the internal rotation profile. This can be mapped from the identification of the modes with $m \neq 0$ once the central $m=0$ component of these modes has been fixed by the models. Adequate seismic modelling of core convection and interior rotation is thus within reach, provided that one succeeds in the identification of at least two, and preferably a much larger set of $(\nu, \ell, m, n)$. This observational requirement demands combined high-precision multicolour photometric and high-resolution spectroscopic measurements with a high duty cycle (typically above 50\%). For an extensive introduction into asteroseismology, its recent successes, and its challenges, I advise the review papers by Kurtz (2006) and by De Ridder (2006). None of the successful cases so far concerns a close binary ...

\section{The specific case of oscillations in close binaries}

Close binary stars have always played a crucial role in astrophysics, not only because, besides pulsating stars, they allow stringent tests of stellar evolution models, but also because they are laboratories in which specific physical processes, which do not occur in single stars, take place. Understanding these processes is important because at least half of all stars occur in multiple systems.

Close binaries are subjected to tidal forces and can evolve quite differently than single stars. Seismic mass and age estimates of pulsating components in close binaries of different stages of evolution, would allow to refine the binary scenarios in terms of energy loss and to probe the interior structure of stars subject to tidal effects in terms of angular momentum transport through non-rigid internal rotation.

\subsection{Overview of observational data}

Two excellent review papers on pulsating stars in binaries and multiple systems (including clusters) are available in Pigulski (2006) and Lampens (2006). These are highly recommended to the reader who wants to get a clear overview of the observational status 
and become familiar with this subfield of binary star research. One learns from these works that numerous pulsating stars are known in binaries, that lots of open questions remain concerning the confrontation between tidal theory and observational data, and that the best cases to monitor in the future are pulsating stars in eclipsing binaries. Eclipsing binaries have indeed revealed values for the core extent in B stars in excess of those found from asteroseismology of single B stars (Guinan et al. 2000). A natural thing to do would be to repeat the type of asteroseismic studies that led to the core overshoot value and internal rotation profile of single stars, as discussed in the previous section, but then for pulsating stars in eclipsing binaries. This would allow to disentangle the core overshoot from the internal rotation with higher confidence level than for single stars. In this respect, I refer to Pigulski et al., Golovin \& Pavlenko, and Latkovic (these proceedings) for new discoveries of pulsating stars in eclipsing binaries and to Brüntt et al. (these proceedings) for the best quality data available of such systems to date.

\subsection{Mode identification through eclipse mapping}

A remark worth giving here is the potential to perform mode identification through the technique of eclipse mapping in eclipsing binaries with a pulsating component. This idea was put forward more than 30 years ago by Nather \& Robinson (1974), who interpreted the phase jumps of $360^{\circ}$ in the nova-like binary UX UMa in terms of non-radial oscillation modes of $\ell=2$. We now know that this interpretation was premature and that the observed phase phenomenon is far better explained in terms of an oblique rotator model.

Mkrtichian et al. (2004) excluded odd $\ell+m$ combinations for the Algol-type eclipsing binary star AS Eri from the fact that the disk-integrated amplitude disappears during the eclipse. Gamarova et al. (2005) and Rodríguez et al. (2004) made estimates of the wavenumbers for the Algol-type eclipsing binaries AB Cas and found a dominant radial mode, in agreement with the out-of-eclipse identification. By far the best documented version of mode identification from photometric data using eclipse mapping is available in Reed et al. (2005). While their primary goal was to search for evidence of tidally tipped pulsation axes in close binaries, they also made extensive simulations, albeit for the very specific case of eclipse mapping of pulsating subdwarf B-star binaries. They find that $\ell>2$ modes become visible during an eclipse while essentially absent outside of eclipse. Their tools have so far only been applied to the concrete cases of KPD 1930+2752 and of PG 1336-018 (Reed et al. 2006) but without clear results.

We must conclude that, still today, more than 30 years after the original idea, mode identification from eclipse mapping is hardly applied successfully in practice, and it certainly has not been able to provide constraints on the wavenumbers for stars which have been modelled seismically. New promising work along this path is, however, in progress (Mkrtichian, private communication).

\subsection{Pressure versus gravity modes}

One thing to keep in mind is that there are two types of oscillations from the viewpoint of the acting forces, and that only one type is relevant in the context of tidal excitation. One either has pressure modes, for which the dominant restoring force is the pressure, or gravity modes, for which the dominant restoring force is buoyancy. Tidal excitation can only occur whenever the orbital frequency is an integer multiple of the pulsation frequency, the integer typically being smaller than ten. This follows from the expression of the tide-generating gravitational potential (e.g., Claret et al. 2005, and references therein). Moreover, in that case, one expects only $\ell=2$ modes to be excited, with an $m$-value that provides the good combination between the rotation, oscillation and orbital frequencies to achieve a non-linear resonance. Such a situation is much more likely to 
occur for gravity modes, which have, in main-sequence stars, oscillation periods of order days, than for pressure modes which have much shorter periods of hours. The same holds true for compact oscillators, whose pressure modes have short periods of minutes while their gravity modes have periodicities of hours and these may be of the same order as the orbital periods.

Tidal forces can, of course, alter the free oscillations excited in components of binaries. In that case, one expects to see shifts of the frequencies of the free oscillation modes with values that have something to do with the orbital frequency. This alteration can, but does not need to be, accompanied with ellipsoidal variability. In the latter case, the tides have deformed the oscillator from spherical symmetry, and one needs to take into account this deformation in the interpretation of the oscillation modes.

\subsection{Sample studies}

Soydugan et al. (2006) have presented a sample study of 20 eclipsing binaries with a $\delta$ Sct-type component. They came up with a linear relation between the pulsation and orbital period:

$$
P_{\text {puls }}=(0.020 \pm 0.002) P_{\text {orb }}-(0.005 \pm 0.008) .
$$

This observational result implies that tidal excitation cannot be active in this sample of binaries, because this would demand a coefficient larger than typically 0.1 as mentioned above, i.e., an order of magnitude larger than the observed one.

A similar conclusion was reached by Fontaine et al. (2003), who investigated if the oscillations in pulsating subdwarf B stars could be tidally induced, given that $2 / 3$ of such stars are in close binaries. Tidal excitation can, at most, explain some of the gravity modes observed in some such stars but this is not yet proven observationally. On the other hand, all the formation channels for subdwarf B stars involve close binary evolution (Han et al., Pulstylnik \& Pustynski, Morales-Rueda et al., these proceedings). An asteroseismic high-precision mass and age estimate of such a star would imply stringent constraints on the proposed scenarios and on the role of the binarity for the oscillatory behaviour (see $\mathrm{Hu}$ et al., these proceedings).

Aerts \& Harmanec (2004), finally, made a compilation of some 50 confirmed line-profile variables in close binaries (mainly OBA-type stars). They could not find any significant relation between the binary and variability parameters of these stars.

We come to the important conclusion that we have by no means a good statistical understanding of the effects of binarity on the components' oscillations. This situation can only be remedied by performing several case studies of pulsating close binaries in much more depth than those existing at present.

\section{Towards successful seismic modelling of binaries}

In general, we have to make a distinction between three different situations when studying oscillations in close binaries with the goal to make seismic inferences of the stellar structure.

\subsection{Reduction of the error box of fundamental parameters}

In a first case, the binarity is simply an asset for the asteroseismologist, because it allows for a reduction of the observational error box of the fundamental parameters of the pulsating star. This case is relevant whenever we lack an accurate parallax value, and thus a good estimate of the luminosity and the mass. This is mainly the case for OBtype stars, but sometimes also for cooler stars or compact objects. In the absence of a 
good mass or luminosity estimate, the asteroseismologist cannot discriminate sufficiently between the seismic models fulfilling the observed and identified oscillation modes. It was recently shown that a combined observational effort based on interferometry and highprecision spectroscopy can enhance significantly the precision of luminosity and mass estimates in double-lined spectroscopic binaries with a pulsating component (Davis et $a l$. , these proceedings), if need be after spectroscopic disentangling as in Ausseloos et al. (2006). Such spectroscopy is in any case also needed to derive the oscillation wavenumbers $(\ell, m)$ (e.g., Briquet \& Aerts 2003, Zima 2006).

The best case study of a pulsating star whose binarity was of great help in the seismic interpretation is the one of $\alpha$ Cen $\mathrm{A}+\mathrm{B}$ with two pulsating components (Miglio \& Montalban 2006, and references therein). The binarity gave such stringent constraints in this case, that in-depth information on the interior of both components was found. In particular, it was found that the components seem to have different values of the mixing length parameter and that the primary, being of the same spectral type as the Sun, is right at the limit of having or not a small convective core. The seismic analysis also provided an accurate age estimate of the system. We refer to Miglio \& Montalban (2006), and references therein, for the latest results and an overview of the stellar modelling.

Other well-known, but less successful examples are the $\delta$ Sct star $\theta^{2}$ Tau (Breger et al. 2002, Lampens et al. these proceedings) and the B-type pulsators $\beta$ Cen (Ausseloos et al. 2006), $\lambda$ Sco (Tango et al. 2006) and $\psi$ Cen (Bruntt et al. 2006). For these four stars, which are all fairly rapid rotators, our comprehension of the observed pulsational behaviour is at present insufficient for detailed seismic inference of their interior structure. In particular, we lack reliable mode identification of the detected frequencies. Not being able to identify the oscillation modes properly is the largest stumbling block in asteroseismology of single stars as well.

\subsection{Tidal perturbations of free oscillations}

The second case concerns seismic targets whose free oscillation spectrum is affected by the tides. The first such situation was reported for the $\delta$ Sct star 14 Aur A, a 3.8-d circular binary whose close frequency splitting of an $\ell=1$ mode does not match the one of a single rotating star and was interpreted in terms of a tidal effect by Fitch \& Wisniewski (1979). Alterations of the free oscillation modes by tidal effects have also been claimed for the three $\beta$ Cep stars $\alpha$ Vir (also named Spica) which has an orbital period $P_{\text {orb }}=4.1 \mathrm{~d}$ and an eccentricity $e=0.16$ (Aufdenberg, these proceedings, Smith 1985a,b), $\sigma$ Sco with $P_{\text {orb }}=33 \mathrm{~d}, e=0.44$ (Goossens et al. 1984) and $\eta$ Ori Aab with $P_{\text {orb }}=8 \mathrm{~d}, e=0.01$ (De Mey et al. 1996).

When, besides oscillation frequencies, the orbital frequency and its harmonic is found in the frequency spectrum, one is dealing with oscillations in an ellipsoidal variable. This case occurs for the $\beta$ Cep stars $\psi^{2}$ Ori with $P_{\text {orb }}=2.5 \mathrm{~d}, e=0.05$ (Telting et al. 2001) and $\nu$ Cen with $P_{\text {orb }}=2.6 \mathrm{~d}, e=0$ (Schrijvers \& Telting 2002), as well as for the $\delta$ Sct stars $\theta$ Tuc with $P_{\text {orb }}=7 \mathrm{~d}, e=0$ (De Mey et al. 1998), XX Pyx with $P_{\text {orb }}=1.2 \mathrm{~d}, e=0$ (Aerts et al. 2002) and HD 207251 with $P_{\text {orb }}=1.5 \mathrm{~d}, e=0$ (Henry et al. 2004). The deformation of the pulsator has so far been neglected in the interpretation of the oscillation frequencies in these binaries. The reason is clear: the complexity of the mathematical description of non-radial oscillations for a deformed star is huge compared to the case of a sphericallysymmetric star. Aerts et al. (2002) have pointed out that this omission may well be the reason why the extensive efforts to model XX Pyx seismically, as in Pamyatnykh et al. (1998), have failed so far. 


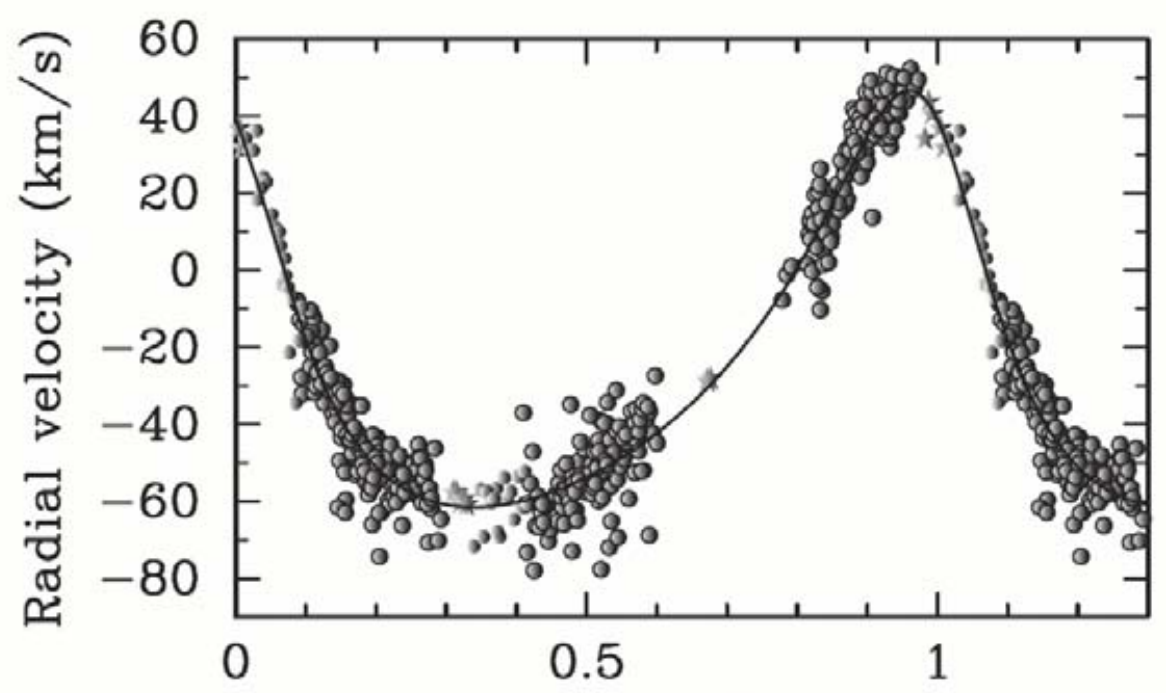

\section{Orbital Phase}

Figure 1. Observed radial velocities of HD 209295 (symbols) phased with the orbital solution (full line). For an explanation of the symbols, we refer to Handler et al. (2002), from which this figure was reproduced with permission from MNRAS.

\subsection{Tidally-excited oscillations}

There are at present only two cases known that meet the requirement of having tidallyexcited oscillation modes. It concerns the slowly pulsating B star HD 177863 (B8V), for which De Cat et al. (2000) found an oscillation mode whose pulsation frequency is an exact multiple of 10.00 times the orbital frequency. The orbit of the star is very eccentric with $e=0.77$ which provides a good situation to achieve a resonance between an $\ell=2$ mode with a period of 1 day and the orbit of 10 days. Willems \& Aerts (2002) made computations based on tidal oscillation theory for this star and indeed came up with the possibility that it is undergoing a non-linear resonantly excited $\ell=2$ oscillation mode. This star has only one confirmed oscillation so far, such that seismic modelling is not yet possible since this requires at least two well-identified modes.

By far the most interesting case of tidally-induced oscillations was found by Handler et al. (2002). They discovered the star HD 209295 (A9/F0V) to be a binary with $P_{\text {orb }}=$ $3.11 \mathrm{~d}$ and $e=0.352$ (see Figure 1) exhibiting one $\delta$ Sct-type pressure mode and nine $\gamma$ Dor-type gravity modes, of which five have an oscillation frequency which is an exact multiple of the orbital frequency. The frequency spectra of the star, after subsequent stages of prewhitening, are shown in Figure 2. The authors also predicted frequencies of tidally-excited modes for appropriate fundamental parameters of the primary and found several such gravity modes to agree with the observed ones, after having corrected the latter for the surface rotation of the star (see Figure 3). The unfortunate situation of not being able to identify the modes occurs again for this star, such that seismic tuning of its structure was not achieved so far.

\section{Conclusions and outlook}

We recall that the requirements for successful seismic modelling of a star are stringent. We need accurate frequency values, reliable identification of the spherical wavenumbers 

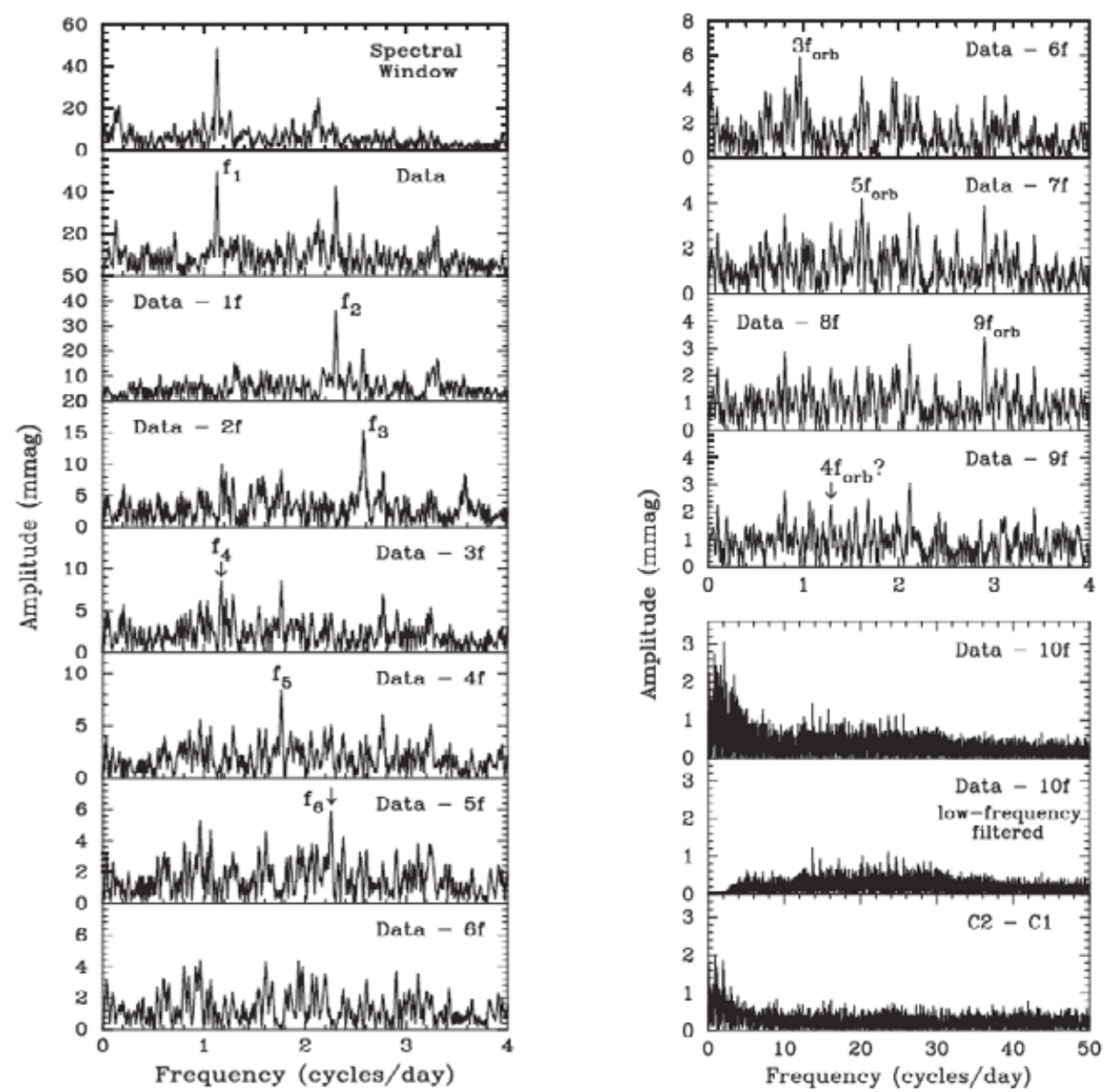

Figure 2. Left: Spectral window and amplitude spectra of the $B$ filter time-series photometry of HD 209295 after subsequent stages of prewhitening. Top Right: amplitude spectra of the combined $B$ and scaled $V$ filter data, after prewhitening with the 6 -frequency solution shown in the left panel. Several harmonics of the orbital frequency are found. Bottom Right: amplitude spectra of the differential magnitudes of the comparison stars. Figure reproduced from Handler et al. (2002) with permission from MNRAS.

$(\ell, m)$ and accurate fundamental parameters with a precision better than typically $10 \%$ before being able to start the modelling process. These requirements demand long-term high signal-to-noise and time-resolved spectroscopy and multicolour photometry with a duty cycle above, say, 50\%. It is important that the data cover the overall beat-period of the oscillations, ranging from a few days for compact oscillators up to several months for main-sequence gravity-mode oscillators. These requirements have been met recently for a few bright single stars, with impressive improvement for their interior structure modelling, but not yet for a pulsating star in a close binary. For those candidates that came close to meeting these requirements, the problem of mode identification occurred and prevented seismic tuning.

We come to the conclusion that the potential of seismic modelling of close binaries is extremely good, particularly for eclipsing binaries. At the same time, its application is extremely demanding from an observational point of view. It is clear that efficient 


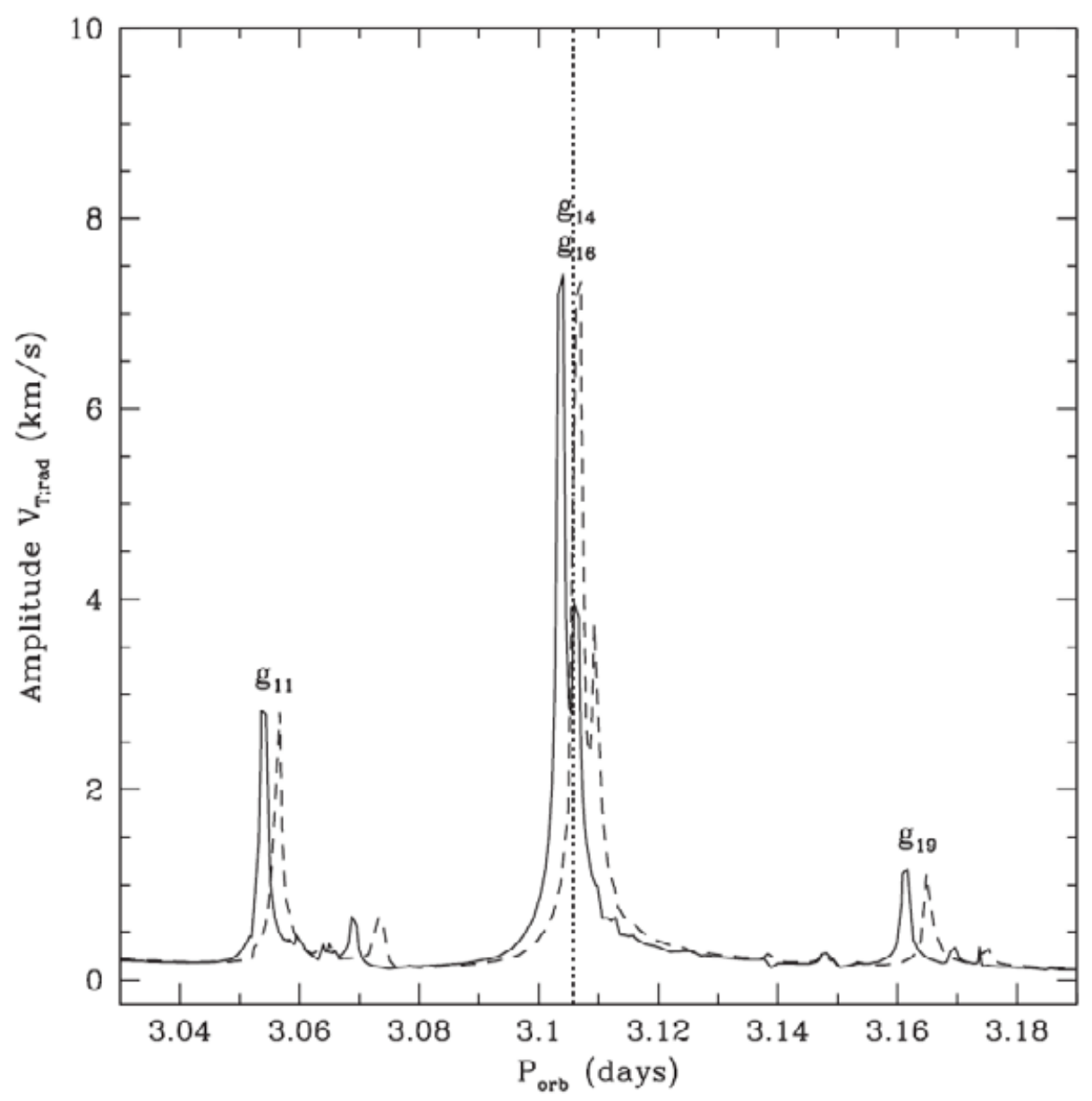

Figure 3. Predicted amplitudes of tidally induced radial velocity variations in a model for HD 209295, for two slightly different values of the rotation frequency $\left(1.852 \mathrm{~d}^{-1}\right.$ : full line; $1.854 \mathrm{~d}^{-1}$ : dashed line). The orbital frequency of the star is indicated by the dotted vertical line. Figure reproduced from Handler et al. (2002) with permission from MNRAS.

progress in this field can only be achieved from coordinated multisite multitechnique observing campaigns, preferrably in combination with uninterrupted space photometry. I strongly encourage the binary and asteroseismology communities to collaborate and take up this challenging project.

\section{Acknowledgements}

The author is much indebted to the Research Council of the Catholic University of Leuven for significant support during the past years under grant GOA/2003/04. She is also grateful to the organisers of this meeting for the opportunity to present this work and to Petr Harmanec for inspiring discussions and wise lessons on binary stars.

\section{References}

Aerts, C., Handler, G., Arentoft, T., Vandenbussche, B., Medupe, R., \& Sterken, C. 2002, MNRAS 333, L35 
Aerts, C. \& Harmanec, P. 2004, in: R. W. Hidlitch, H. Hensberge \& K. Pavlovski (eds.), Spectroscopically and Spatially Resolving the Components of the Close Binary Stars, ASPC (ASP: San Francisco), vol. 318 , p. 325

Aerts, C., Thoul, A., Dasźynska, J., Scuflaire, R., Waelkens, C., Dupret, M-A., Niemczura, E., \& Noels, A. 2003, Science 300, 1926

Asplund, M., Grevesse, N., Sauval, A. J., Allende Prieto, C., \& Kiselman, D. 2005, A 8 A 435, 339

Ausseloos, M., Aerts, C., Lefever, K., Davis, J., \& Harmanec, P. 2006, A\&̈A 455, 259

Breger, M., Pamyatnykh, A. A., Zima, W., Garrido, R., Handler, G., \& Reegen, P. 2002, MNRAS 336,249

Briquet, M. \& Aerts, C. 2003, A\&A 398, 687

Bruntt, H., Southworth, J., Torres, G., Penny, A. J., Clausen, J. V., \& Buzasi, D. L. 2006, Aש̋A, 456,651

Claret, A., Giménez, A., Zahn, J.-P. (eds.) 2005, Tidal Evolution and Oscillations in Binary Stars, ASPC (ASP: San Francisco), vol. 333

Christensen-Dalsgaard, J. 2002, Rev. Modern Phys. 74, 1073

De Cat, P., Aerts, C., De Ridder, J., Kolenberg, K., Meeus, G., \& Decin, L. 2000, AछA 355, 1015

De Mey, K., Aerts, C., Waelkens, C., \& Van Winckel, H. 1996, A\& A 310, 164

De Mey, K., Daems, K., \& Sterken, C. 1998, A\&A 336, 527

De Ridder, J. 2006, in: C. Sterken, \& C. Aerts (eds.), Astrophysics of Variable Stars, ASPC (ASP: San Francisco), vol. 349, p. 129

Fitch, W. S. \& Wisniewski, W. Z. 1979, ApJ 231, 808

Fontaine, G., Brassard, P., Charpinet, S., Green, E. M., \& Willems, B. 2003, in: M.J. Thompson, M.S. Cunha, M.J.P.F.G. Monteiro (eds.), Asteroseismology Across the HR Diagram, ApSS (Kluwer Academic Publishers), vol. 284, p. 517

Gamarova, A. Yu., Mkrtichian, D. E., \& Rodríguez, E. 2005, in: A. Claret, A. Giménez \& J.-P. Zahn (eds.), Tidal Evolution and Oscillations in Binary Stars, ASPC (ASP: San Francisco), vol. 333 , p. 258

Goosens, M., Lampens, P., de Maerschalck, D., \& Schrooten, M. 1984, A\&A 140, 223

Guinan, E. F., Ribas, I., Fitzpatrick, E. L., Giménez, A., Jordi, C., McCook, G. P., \& Popper, D. M. 2000, ApJ 544, 409

Handler, G., Balona, L. A., Shobbrook, R. R., Koen, C., Bruch, A., Romero-Colmenero, E., Pamyatnykh, A. A., Willems, B., Eyer, L., \& James, D. J., Maas, T. 2002, MNRAS 333, 262

Henry, G. W., Fekel, F. C., \& Henry, S. M. 2004, AJ 127, 1720

Kurtz, D.W. 2006, in: C. Sterken, \& C. Aerts (eds.), Astrophysics of Variable Stars, ASPC (ASP: San Francisco), vol. 349, p. 101

Lampens, P. 2006, in: C. Sterken, \& C. Aerts (eds.), Astrophysics of Variable Stars, ASPC (ASP: San Francisco), vol. 349, p. 153

Maeder A. \& Meynet G. 2000, ARA\&A 38, 143

Mazumdar, A., Briquet, M., Desmet, M., \& Aerts, C. 2006, $A \mathscr{\varepsilon} A$, in press

Miglio, A. \& Montalban, J. 2005, A\& $A 441,615$

Mkrtichian, D. E., Kusakin, A. V., Rodriguez, E., Gamarova, A. Yu., Kim, C., Kim, S.-L., Lee, J. W., Youn, J.-H., Kang, Y. W., Olson, E. C., \& Grankin, K. 2004, A\&̛A 419, 1015

Nather, R. E. \& Robinson, E. L. 1974, ApJ 190, 637

Pamyatnykh, A. A., Handler, G., \& Dziembowski, W. A. 2004, MNRAS 350, 1022

Pamyatnykh, A. A., Dziembowski, W. A., Handler, G., \& Pikall, H. 1998, A\&6A 333, 141

Pigulski, A. 2006, in: C. Sterken, \& C. Aerts (eds.), Astrophysics of Variable Stars, ASPC (ASP: San Francisco), vol. 349, p. 137

Reed, M. D., Brondel, B. J., \& Kawaler, S. D. 2005, ApJ 634, 602

Reed, M. D.\& Whole Earth Telescope Xcov 21 and 23 Collaborations 2006, Memorie della Societa Astronomica Italiana 77, 417

Rodríguez, E., García, J. M., Gamarova, A. Y., Costa, V., Daszyńska-Daszkiewicz, J., LópezGonzález, M. J., Mkrtichian, D. E., \& Rolland, A. 2004, MNRAS 353, 310

Schrijvers, C. \& Telting, J. H. 2002, A\&A 394, 603 
Smith, M.A. 1985a, ApJ 297, 206

Smith, M.A. 1985b, ApJ 297, 224

Soydugan, E., Ibanoğlu, C., Soydugan, F., Akan, M. C., \& Demircan, O. 2006, MNRAS 366, 1289

Tango, W. J., Davis, J., Ireland, M. J., Aerts, C., Uytterhoeven, K., Jacob, A. P., Mendez, A., North, J. R., Seneta, E. B., \& Tuthill, P. G. 2006, MNRAS 370, 884

Telting, J. H., Abbott, J. B., \& Schrijvers, C. 2001, A\&\&A 377, 104

Unno, W., Osaki, Y., Ando, H., Saio, H., \& Shibahashi, H. 1989, Nonradial Oscillations of Stars, 2nd Edition, University of Tokyo Press

Willems, B. \& Aerts, C. 2002, A\&A 384, 441

Zima, W. 2006, A\&广A 455, 227

\section{Discussion}

Robert Wilson: Have you looked into the time scale for orbital changes caused by dissipation of orbital energy by excitation of pulsations?

AERTS: I haven't, but other peoples did. In any case, such energy dissipation happens on an evolutionary timescale. This is very different from apsidal motion, with typical timescales between 10 to 100 years (see talk by Gimenez). The computation of timescales of energy dissipation through oscillations (i.e. in the case where there is no mass transfer) is difficult, because it is very dependent on the assumed initial conditions, in particular on the rotation velocity. Analytical computations of energy loss are availabe in, e.g., Willems et al. (2003, A\&A, 397, 973). Numerical computations were made by, e.g., Witte \& Savonije (2002, $A \mathscr{E} A, 386,222)$. All these works, however, predict too long timescales if we compare them with those derived from data of high-mass X-ray binaries (the only observed cases available). This might be due to the much stronger radiative damping during resonances than anticipated so far. Besides the orbital energy loss, one also has to keep in mind that the oscillations probably imply significant angular momentum loss of the components through non-rigid internal rotation.

PATRicia LAmpens: How much time was needed to reach the significant new insight for single stars from the oscillations that you discussed (non-rigid internal rotation and estimate of core overshoot), given that the combination of pulsation and binarity is even more demanding?

AERTS: We managed to derive the non-rigid internal rotation in two main-sequence B stars so far. These stars have multiple oscillation periods of the order of several hours. One star was monitored with one and the same instrument from a single site during 21 years! The other one was monitored during a well-coordinated multisite photometric and spectroscopic campaign lasting 5 months and involving about 50 observers (see references in the paper). It is therefore clear that asteroseismic inference requires longterm monitoring. I think that the binarity does not impose the necessity of even longer runs (at least not for close binaries), because the most stringent demand is the frequency accuracy and the mode identification, while the orbital determination will result naturally and efficiently from a multsite spectroscopic effort. 